\title{
Surgical treatment of retrorectal tumors: our experience in 34
} patients

\author{
Saverio Coiro, Domenico Spoletini, Fabio Giorgiano, Aldo Nunziale, \\ Elena Manna, Francesca De Lucia and Giuseppe Pappalardo*
}

\author{
Address: Department of General Surgery, Surgical Specialities and Organ Transplantation "P. Stefanini", Umberto I Hospital "Sapienza" University \\ of Rome, Italy \\ * Corresponding author
}

\author{
from XXI Annual Meeting of The Italian Society of Geriatric Surgery \\ Terni, Italy. 4-6 December 2008 \\ Published: I April 2009 \\ BMC Geriatrics 2009, 9(SuppI I):A28 doi:10.1 186/I47I-23I8-9-SI-A28
}

This abstract is available from: http://www.biomedcentral.com/|47I-23 I8/9/SI/A28

(c) 2009 Coiro et al; licensee BioMed Central Ltd.

\section{Background}

The retrorectal tumors represent a heterogeneous group of benign and malignant neoplasms which are currently treated without uniform criteria. The most used classifications are based on different histologic origin of these tumors, on their presumed nature (inflammatory or neoplastic), or on their size. A standardization of the surgical approach based on all these characteristics is still now lacking.

\section{Materials and methods}

Between 1989 and 2007 we submitted to surgery 35 patients for retrorectal tumors. Two radiologists, who were blind to the patients records, separately reviewed preoperative CT and MR findings and classified the tumors in three groups: group I those arising in the presacral area, group II those arising in the sacrum or spinal cord growing anteriorly, group III those arising in the sacrum growing posteriorly. The aims were: 1) to plan the surgical approach basing on CT and/or MR findings, particularly the possible involvement of adjacent organs; 2 ) to involve in the surgical planning all the different specialists needed (neurosurgeons, orthopedic surgeons, etc.) to optimize the results, without modifying the surgical program during the procedure.

\section{Results}

CT and MR yielded the information required in the surgical planning in 17 of the 18 (94.5\%) group I tumors, in all $12(100 \%)$ group II tumors and in 4 of $5(80 \%)$ of group III tumors. In all 18 patients of group I intraoperative reports and the histological examination confirmed the correct preoperative classification. The surgical approach had to be changed in 1 patient $(5.5 \%)$ of this group. In all 12 patients of group II the preoperative classification was confirmed by intraoperative reports and histological examination. The preoperative classification was confirmed in 4 of 5 group III $(80 \%)$. In all but one patient the not prevented involving of coccyx needed a change of surgical strategy.

\section{Conclusion}

The diagnostic imaging techniques provide the information needed to successfully plan the surgical intervention in most of the patients with retrorectal tumors (in 33/35 patients - 93\% in our experience). This information is essential in preoperatively involving different surgical specialists and in planning the surgical approach thus allowing an in optimization of the surgical resection. 